BULL. AUSTRAL. MATH. SOC.

VOL. $21(1980), 317-320$.

\title{
ROLE OF COLLECTIVE MODES IN SOME SURFACE PROPERTIES OF METALS
}

\author{
Peter Summerside
}

The theory of the solid state for unbounded bulk samples has, since the advent of quantum mechanics, reached a high degree of sophistication, and the knowledge of the properties of a wide range of solids is adequate for their extensive technological and industrial use. However, the theoretical explanation of many well known phenomena in terms of the electronic properties of solids is far from complete, mainly because of the inherent mathematical difficulties incurred when the ubiquitous surface is introduced. Many properties associated with metal surfaces and interfaces, and of their interaction with external configurations of charges and polarisable molecules and atoms can be understood in terms of the characteristic collective oscillations (bulk and surface plasmons) of their valence or conduction electrons. The use of Bloch's hydrodynamic formalism has facilitated considerable understanding of the properties associated with metal surfaces, to which this thesis makes some further contribution.

In Chapter 1, the hydrodynamic model is defined within the context of the many-body solid-state problem, and the collective oscillations are introduced with a derivation of the hydrodynamic dispersion relations for the surface and bulk plasmons. Throughout, the step-function model for the surface is used and the well known effect of a more realistic density profile on the surface plasmon dispersion frequencies is indicated in this chapter. Chapter 1 also contains a brief discussion on the necessity, within the hydrodynamic model, of the phenomenological introduction of an

Received 12 October 1979. Thesis submitted to the Australian National University, August 1979. Degree approved December 1979. Supervisor: Dr J. Mahanty., 
upper cutoff in the wavenumber for both bulk and surface plasmons.

The finiteness of the velocity of light is a fact of life, and while it may be neglected for interactions over very short distances, retardation is important for dispersion interactions over larger separation distances. In addition, the effect of the transversality of electromagnetic fields has a dramatic effect on the small wavenumber (or momentum) surface plasmons which, while basically of longitudinal nature, also take on a transverse character in this region. Retardation effects are considered in Chapter 2, where the hydrodynamic equation of motion is solved in conjunction with the full set of Maxwell's equations for the general case of a charge source situated between two separated metal half-spaces. This leads, in the appropriate limits, to an evaluation of the retarded dispersion interactions between two identical metals and of a charge (and hence a simple molecule) with a metal surface. The general retarded dispersion relations for the symmetric and antisymmetric surface plasmon branches in the coupled two-metal system are derived and the universal effect of retardation in reducing the van der Waals interactions by increasing their dependence on the inverse separation distance by a power, is clearly indicated in terms of its effect on the surface plasmon dispersion frequencies. The effects of retardation can always be generalised qualitatively, and for simplicity the remaining chapters deal only with the nonretarded limit.

Following a discussion of the role of $v d W$ forces in physisorption, and the question of an appropriate reference plane for their measurement, the explicit contributions from bulk and surface plasmons to the vaW interaction of a molecule with a metal surface are given in the normal mode analysis of Chapter 3. The normal mode analysis is also used to derive the nonretarded dispersion relations for the surface plasmon branches for two identical coupled metal half-spaces, thus demonstrating the role of bulk plasmons in their interaction. The fact that all bulk contributions vanish in the local or spatially nondispersive approximation is the reason for the common inferral that such interactions are of purely surface plasmon origin.

of considerable interest for the practical use of metals is the understanding of the electronic properties of a bimetallic interface. A derivation of the boundary conditions which relax the infinite surface 
barriers at a bimetallic junction is outlined in Chapter 4. The collective modes of the bimetallic system are derived with these relaxed boundary conditions (giving the interface plasmon) and the effect of cleaving and separating the two metals is then considered. This leads to the derivation of 'the generalisations of the upper (symmetric) and lower (antisymmetric) surface plasmon branches well known for identical metals. In addition, a novel character of the electron response, namely the image plasmon, is predicted. It is also argued that, while both the infinite barrier (vanishing normal current) and relaxed (continuous current) boundary conditions lead to an 'interface' mode with the expected infinitewavelength (or zero-wavenumber) behaviour, it is the latter which describes the commonly observed interface mode and hence that this long-established phenomenon has been misunderstood.

The final chapter, Chapter 5, deals with the force on an external charge which is moving with constant velocity parallel to a metal surface. Throughout this chapter the contributions from both bulk and surface plasmons to this dynamical interaction are given in terms of the normal mode decomposition of Chapter 3. The fact that the force components normal and parallel to the particle's motion derive from 'surface' plasmons with phase velocity greater and less than the velocity of the particle, respectively, has been interpreted on the basis of excitation of virtual and real 'surface' plasmons. While complementing this, the origin of the force components is given a much clearer physical basis in terms of the nature (and in fact symmetry properties) of the induced density, which may be written as the sum of a symmetric and an antisymmetric part, again associated with plasmons of 'fast' and slow' phase velocity, respectively, relative to the velocity of the external charge.

Most of the work described in Chapters 2 to 4 has either been published or accepted for publication ([1], [3], [4] and [5]), while the manuscript covering the material presented in Chapter 5 ([2]), has been submitted for publication. 


\section{References}

[1] J. Mahanty and P. Summerside, B.V. Paranjape, "Retarded van der Waals force near a metal surface", Phys. Rev. B (3) 18 (1978), $5174-5176$.

[2] J. Mahanty and $P$. Summerside, "Foxce on a moving charge near a metal surface: effect of spatial dispersion", J. Phys. F (to appear).

[3] P. Summerside, "Plasmon dispersion in two-metal systems", J. Phys. F (8) 9 (1979), 1643-1652.

[4] P. Summerside and J. Mahanty, "Normal mode analysis of the image potential", Solid. State Comm. 28 (1978), 825-828.

[5] P. Summerside and J. Mahanty, "Retarded dispersion interaction between metals", Phys. Rev. B (3) 19 (1979), 2944-2949, 